\title{
Proposal
}

\section{Beam Test of High-performance Hadron Calorimeter for Future Linear Colliders}

\author{
Katsushi Arisaka, ${ }^{a}$ Takashi Asakawa, ${ }^{9}$ Yoshiaki Fujii, ${ }^{b}$ Keitaro Furukawa, \\ Fumiyoshi Kajino, ${ }^{d}$ Teruki Kamon, ${ }^{f 1}$ Naoko Kanaya, ${ }^{c}$ Jun'ichi Kanzaki, ${ }^{b}$ \\ Kiyotomo Kawagoe, ${ }^{c}$ Shin Hong Kim, ${ }^{g}$ Atsuko Nakagawa, ${ }^{g}$ \\ Tatsuro Ohta, ${ }^{g}$ Ryutaro Oishi, ${ }^{g}$ Yoshiyuki Sugimoto, ${ }^{c}$ \\ Tohru Takeshita, ${ }^{e 2}$ Akira Takeuchi, ${ }^{c}$ Satoru Uozumi, ${ }^{g}$ \\ ${ }^{a} U C L A,{ }^{b} K E K,{ }^{c} K o b e$ Univ., ${ }^{d} K o n a n$ Univ., \\ ${ }^{e}$ Shinshu Univ., ${ }^{f}$ Texas A\&M Univ., ${ }^{g}$ Univ. of Tsukuba
}

February 1, 1999

\section{Introduction}

Various efforts of detector $\mathrm{R} \& \mathrm{D}$ for future $e^{+} e^{-}$linear colliders (LC) are extensively made in Japan, in US, and in Europe. A major goal is to design a high-performance detector for a precise study of the Higgs boson and a search for physics beyond the standard model. LCs will provide a very clean experimental environment compared to hadron collider experiments. Detectors with the most excellent performance are indispensable to fully take advantage of its cleanness. For this purpose, we designed and constructed a prototype of a high-performance calorimeter for LCs. The prototype has already been tested at KEK using low-energy beams $(\leq 4 \mathrm{GeV})$, and we need to evaluate its performance at high energies (5-200 GeV). We here submit a proposal for the beam test at FNAL. We would like to carry out the beam test in fall 1999.

\section{Hadron Calorimeter (HCAL)}

\subsection{Main Goal}

One of the most important physics at LCs is discovery and precision study of the Higgs bosons. An intermediate-mass Higgs boson will dominantly be produced via associate production with $Z$-boson, followed by the decay into $b$-quark pair, as

$$
e^{+}+e^{-} \rightarrow Z+H, H \rightarrow b+\bar{b}
$$

This reaction can be most easily detected using a channel in which $Z$ decays into a lepton pair $\left(e^{+} e^{-}\right.$or $\left.\mu^{+} \mu^{-}\right)$. However this channel has a branching fraction of only $7 \%$. Using

\footnotetext{
${ }^{1}$ (Collaboration Representative ; US-side)

${ }^{2}$ (Collaboration Representative; Japan-side)
} 
hadronic decay mode of $Z$ bosons, which has $70 \%$ branching fraction, is indispensable for high-precision study of Higgs boson. Thus $Z Z / W W$ pair production background has to be separated from the signal $(Z H)$ events using a mass reconstruction of two jets.

It is also important to measure the $W$-boson pair creation and the $Z$-boson pair creation separately for a precise measurement of gauge couplings. Thus identification of $W$ and $Z$ bosons through their dijet masses is necessary.

In order to achieve such a performance, the following features are required on calorimeter system :

- Excellent energy resolution and linearity;

- Crack-less structure to measure missing energy reliably;

- Fine-segmented shower-max measurement for precise cluster-track matching and $\gamma / \pi^{0}$ separation;

- Fine granularity for electron/hadron separation and for avoiding accidental overlap of charged tracks and neutral clusters;

- Good time resolution to resolve beam crossing overlap.

One of main goals in this calorimeter prototype is to establish a good energy resolution for hadrons and a crack-less structure.

Assuming a calorimeter of the following energy resolutions, in combination with a central tracker of momentum resolution of $0.01 \% P_{t}$, an extensive simulation study has been carried out.

$$
\begin{aligned}
& \sigma_{E} / E=40 \% / \sqrt{E} \oplus 2 \% \text { for Hadrons } \\
& \sigma_{E} / E=15 \% / \sqrt{E} \oplus 1 \% \text { for EM particles }
\end{aligned}
$$

One of such simulation results is shown in Fig. 1. This shows that an excellent dijet mass resolution can be achieved with a calorimeter of these performances. The shift of the reconstructed mass is due to the failure of track-cluster association, and indicates the importance of fine-granularity. Such calorimeter is expected to be built with a fine-segmented tile-fiber calorimeter of compensated composition.

It has been commonly quoted that a lead-plastic scintillator sampling calorimeter of 4:1 volume ratio achieves so-called compensation, and thus achieves good hadron energy resolution and linearity. However the results of the beam tests of such modules carried out so far have not shown compensation at low energies very well $(e / \pi \sim 1.1$ for $E \leq 10 \mathrm{GeV}$ ). Recently our group carried out a systematic measurement on configuration dependence of various performances at KEK. Some of the results are shown in Fig. 2. The best energy resolution of $33 \% / \sqrt{E}$ was obtained for the $4: 2$ volume ratio, and compensation was found to be achieved at the $9: 2$ volume ratio. What we really need is, however, not the compensation condition itself but excellent energy resolution over wide range of energy. For this purpose beam tests at higher energies are crucial to establish the module performances. 


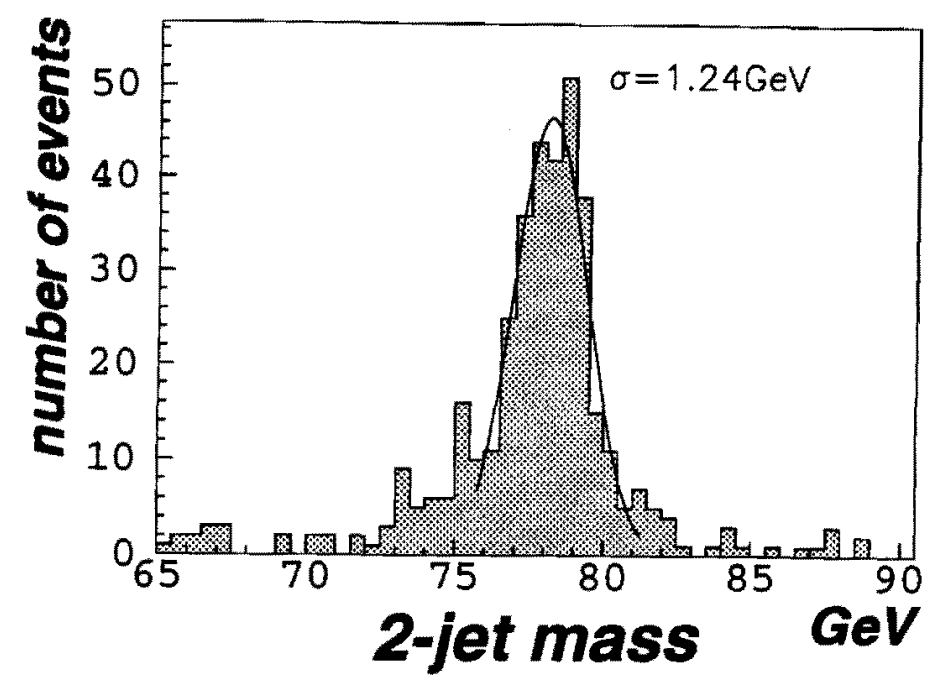

Figure 1: Mass distribution of two jets in decay of a scaler boson. Generated mass and width are $80 \mathrm{GeV}$ and 0 , respectively.
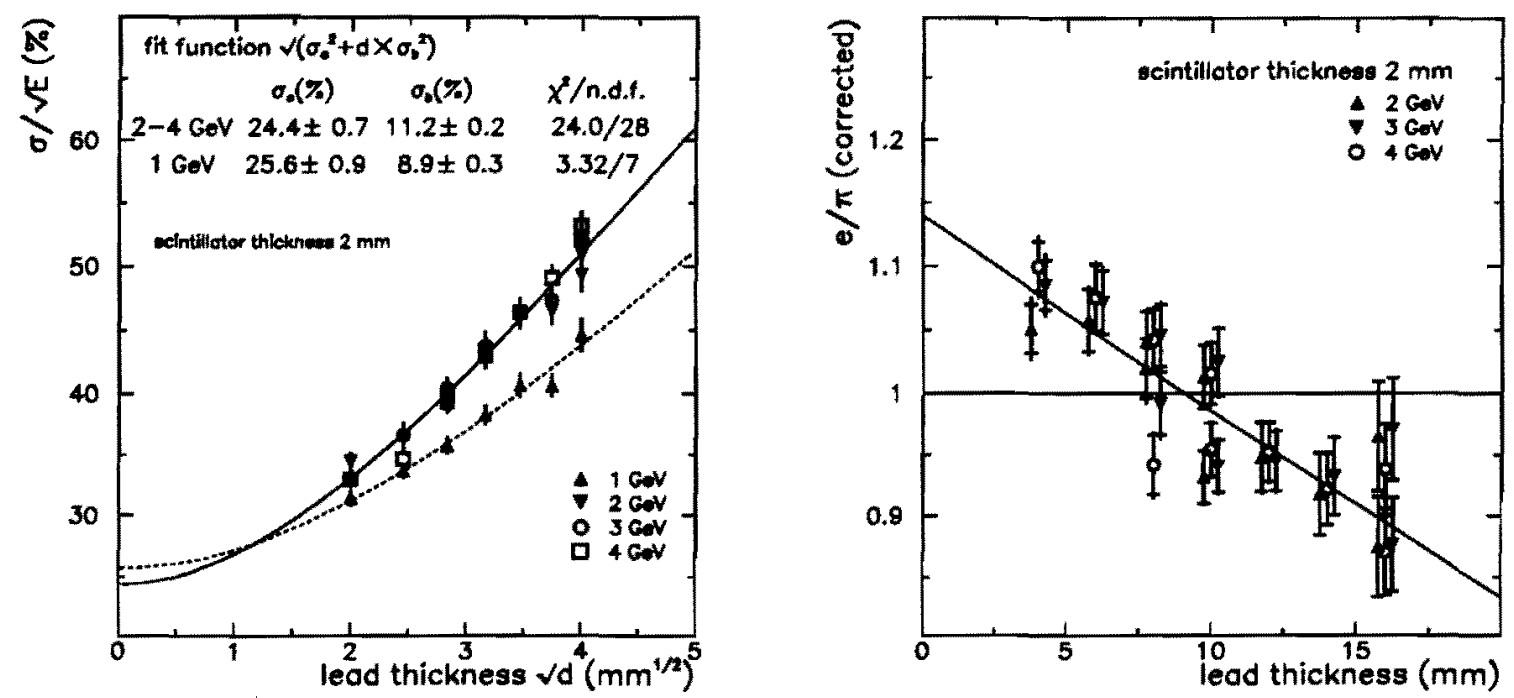

Figure 2: Sampling-frequency dependence of energy resolution for pions (left) and $e / \pi$ ratio (right). 


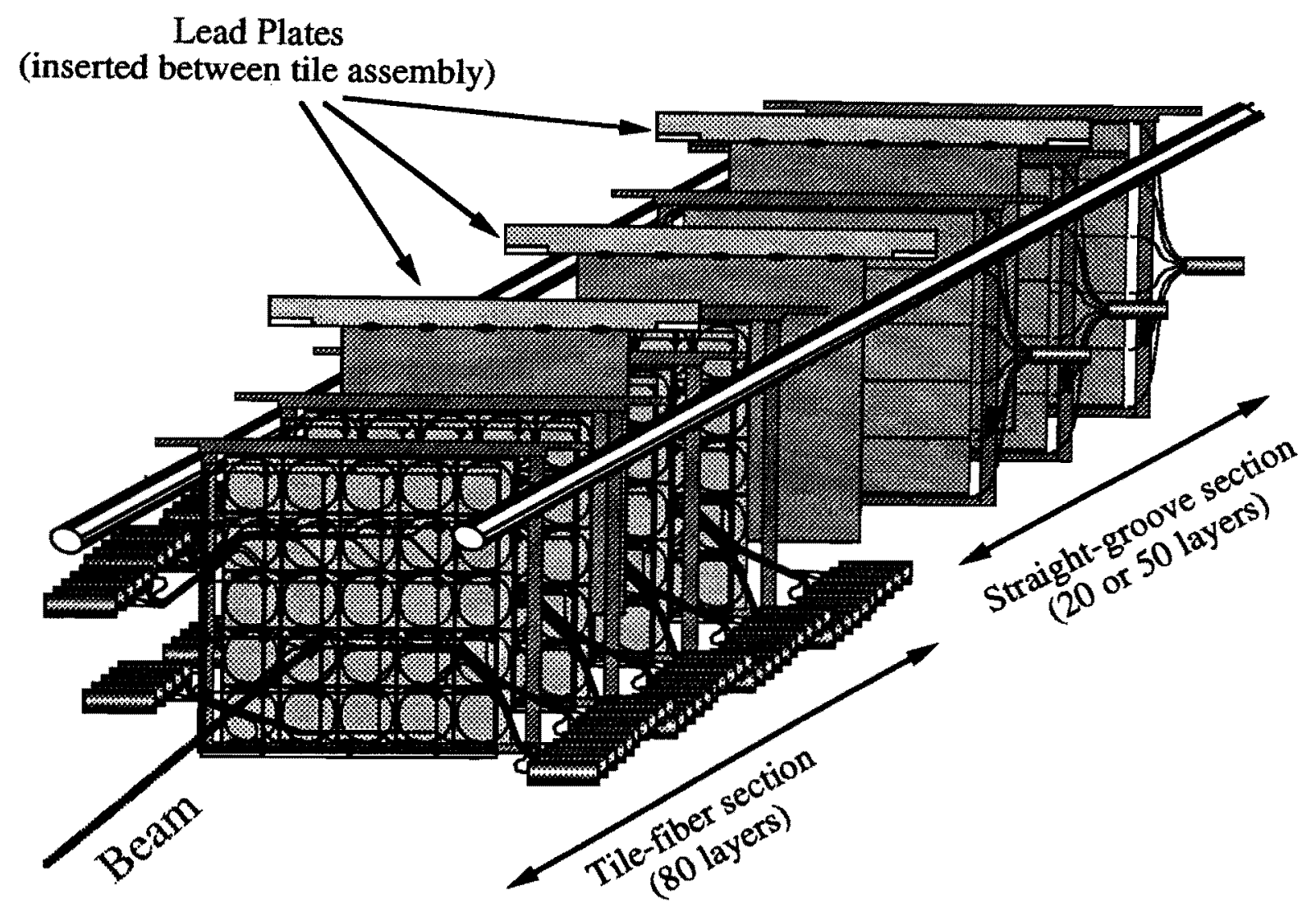

Figure 3: Schematic drawing of hanging-file hadron calorimeter test module.

\subsection{Design}

The prototype calorimeter module is designed based on the tile-fiber technique, which has been extensively studied for the SDC detector at SSC and the CDF detector at Tevatron. This technique enables not only very flexible design for segmentation arrangement but also the best hermeticity, which is essentially important to search for new physics.

A schematic drawing of the test module is shown in Fig. 3. It is a "hangingfile" type test module in which lead plates and scintillator plates are hung over two supporting beams. Each lead plate has dimensions of $100 \mathrm{~cm} \times 100 \mathrm{~cm} \times 0.4 \mathrm{~cm}$. The HCAL assembly can contain 240 lead plates at maximum. There are two types of the scintillator plates; tile-fiber type and straight-groove type. A schematic drawing of each type is shown in Fig. 4. Both types have dimensions of $100 \mathrm{~cm} \times 100 \mathrm{~cm}$, and thickness of the scintillator plates is $0.2 \mathrm{~cm}$. The tile-fiber assembly has a $5 \times 5$ tower structure, while the straight-groove assembly has no transverse segmentation.

The first 80 layers of HCAL are tile-fiber/lead sandwiches, and the remaining part consists of straight-groove/lead sandwiches. Number of the straight-groove/lead layers differs configuration by configuration. Twenty layers of the tile-fiber assembly are optically ganged, and as a result, the tile-fiber part has four longitudinal segmentations in addition to the $5 \times 5$ tower structure. In the straight-groove part, 5 layers are optically 

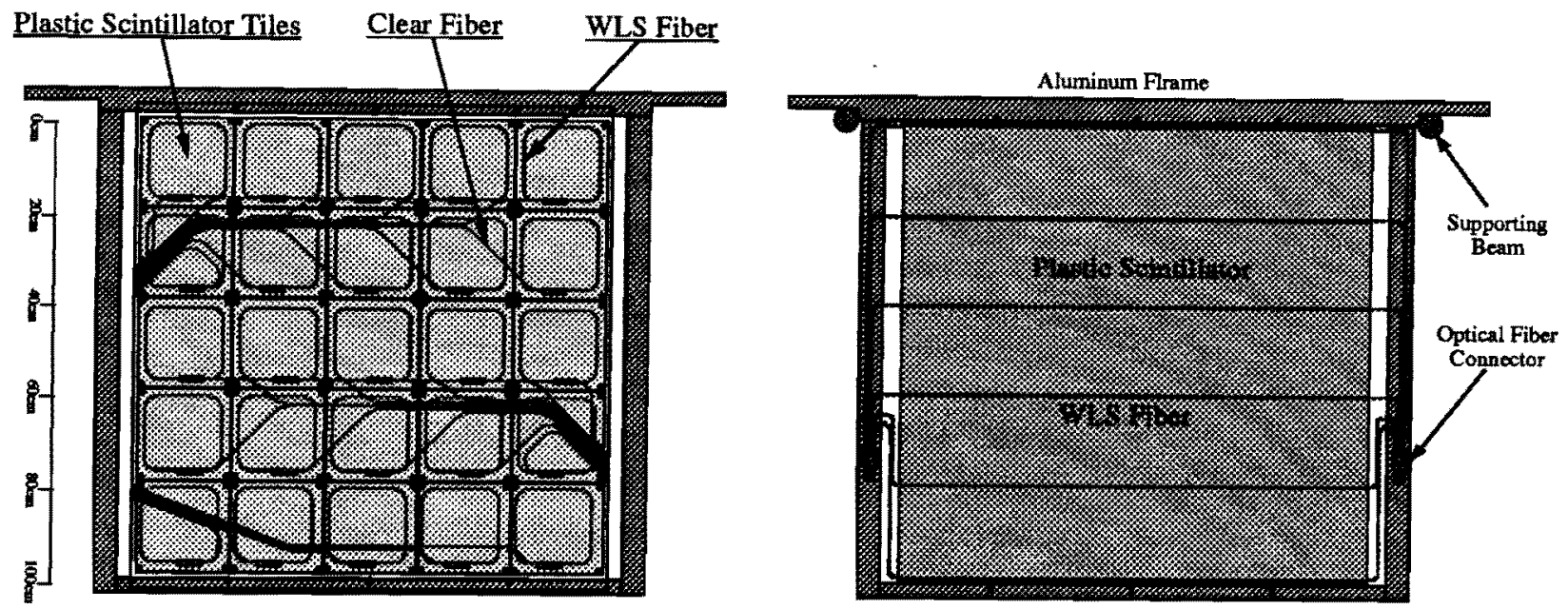

Figure 4: Design of two tile assemblies. The left drawing indicates the tile-fiber assembly, and the right drawing shows the straight-groove assembly.

ganged.

These lead plates and scintillator plates can be arranged on the supporting beams in any order, and thus provide module configuration of various sampling ratios. In the beam test at KEK, the thickness of each layer of the lead plates was varied from $4 \mathrm{~mm}$ to $16 \mathrm{~mm}$ at a step of $2 \mathrm{~mm}$ using $2 \mathrm{~mm}$-thick additional lead sheets. In the beam test at FNAL, however, we are going to study only two configurations (8:2 and 12:2 volume ratios). Performances at 9:2 ratio, at which compensation is achieved, will be obtained by interpolating performances at 8:2 and those at 12:2. Table 1 is a summary of two calorimeter configurations.

\section{Plan}

\subsection{Test Beam Area}

This test module will be placed on a moving stage located at the Test Beam Area E-741. The layout is shown in Fig. 5. This moving stage is needed to inject the beam at the center of each tower to determine calibration constants.

The area of the moving stage is large enough, and our test module can sit on the stage stably as seen in Fig. 5. According to the drawings, the stage and the driving screws have sufficient strength to support the test module, which weighs about 20 metric-tons in total. On the other hand, the driving power of the motor was not identified from the drawing yet. This must be clarified quickly. Replacement of the motor could be necessary.

Another necessary modification is removal of the additional supporting beams on the top of the stage. As shown in Fig. 6, the upper-most towers can not be aligned on the beam line even if the stage comes to the lowest position. Removal of the additional beams can lower the calorimeter module by $16 \mathrm{~cm}$ more, and thus the upper-most towers 
Table 1: Parameters of scintillator plates

\begin{tabular}{lcc}
\hline & Tile-fiber part & Straight-groove part \\
\hline \hline $8: 2$ Configuration & & 50 \\
Total number of layers & 80 & 5 \\
Size of each scintillation plate & $20 \mathrm{~cm} \times 20 \mathrm{~cm} \times 0.2 \mathrm{~cm}$ & $100 \mathrm{~cm} \times 100 \mathrm{~cm} \times 0.2 \mathrm{~cm}$ \\
Number of towers & $25(5 \times 5)$ & 1 \\
Number of longitudinal segmentations & 4 & 10 \\
Thickness of lead plates & $8 \mathrm{~mm}$ & $8 \mathrm{~mm}$ \\
\hline \hline 12:2 Configuration & & \\
Total number of layers & 80 & 20 \\
Size of each scintillation plate & $20 \mathrm{~cm} \times 20 \mathrm{~cm} \times 0.2 \mathrm{~cm}$ & $100 \mathrm{~cm} \times 100 \mathrm{~cm} \times 0.2 \mathrm{~cm}$ \\
Number of towers & $25(5 \times 5)$ & 1 \\
Number of longitudinal segmentations & 4 & 4 \\
Thickness of lead plates & $12 \mathrm{~mm}$ & $12 \mathrm{~mm}$ \\
\hline
\end{tabular}

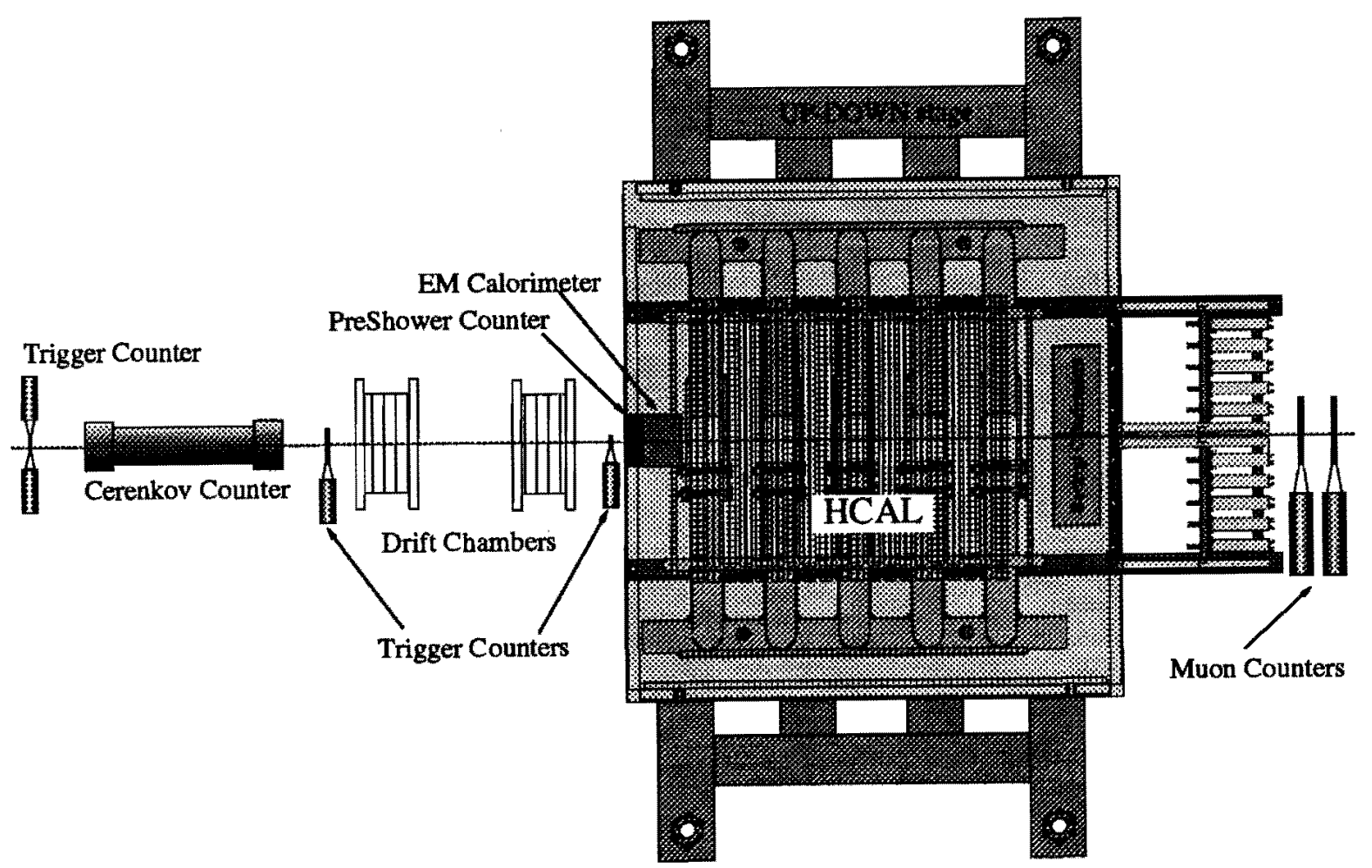

Figure 5: Horizontal view of the layout of the detectors in the experimental hall. Detector sizes/locations are not in scale. 


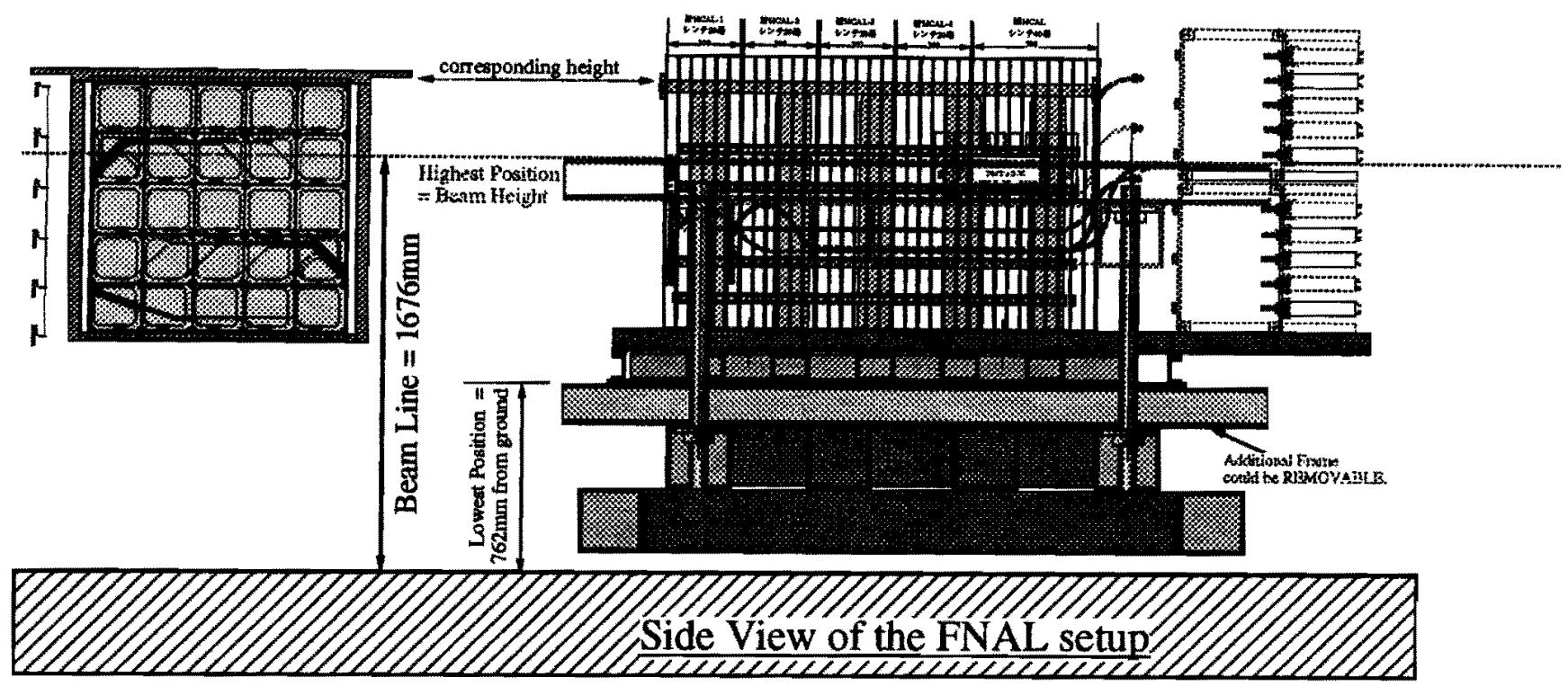

Figure 6: Vertical view for the layout of the detector in the experimental hall. Also shown in the left is a vertical location of towers seen from the front-side when the stage is set to its lowest position.

can be aligned on the beam line.

Horizontal rotation range of the stage is wide enough to inject the beam to each tower.

\subsection{Detector Configuration}

In the beam test at FNAL, we are going to study two configurations (8:2 and 12:2 volume ratios) as described previously. The $2 \mathrm{~mm}$-thick lead sheets, which was used in the experiment at KEK, will not be used at FNAL because handling is extremely difficult.

In addition to the $\mathrm{HCAL}$, we will put appropreate modules as a pre-shower detector, a shower-max detector, and an EM calorimeter in front of HCAL to provide additional particle identification capability. Back-up calorimeters will be also placed downstream of the HCAL to determine the shower leakage. Muon taggers will also be placed at the most downstream.

\subsection{Measurements}

The following performances for two different volume ratios will be studied:

1. Energy resolution;

2. Electron/pion pulse-height ratio (so-called compensation ratio);

3. Linearity; 
4. Position dependence of the responses.

For these measurements, we need electron and pion beams at energies from $5 \mathrm{GeV}$ to $200 \mathrm{GeV}$. It is known that intensity of the beams at energies below $10 \mathrm{GeV}$ are unstable. However, measurements at $5 \mathrm{GeV}$ are just to compare with results obtained at KEK test. Thus the best stability of the beam may not be needed for such low energies.

Non-uniformity of the tile-fiber tower structure has been studied by the CDF group extensively, and an excellent uniformity has been already shown. However thickness of the scintillator plates in our module is much thinner than that in the CDF case in order to achieve compensation ratio. Non-uniformity is thus expected to be bigger in our modules, and response-mapping should be done more precisely.

\subsection{Beam Time}

According to the beam property reports, we can assume that the beam intensity is high enough for our test purpose. The necessary time for the beam test is thus determined by our DAQ system. Our present system achieves DAQ rate of about 30 events/sec. Averaging this rate according to the time structure of the beam spill, we will be able to take data with about 10 events/sec. Based on this DAQ rate, and also taking the parameterchange time into account, necessary beam time for each configuration is estimated as follows:

- Calibration-I (at the beginning): 20 hours $2.5 \mathrm{k}$ events $(0.2 \mathrm{~h})$ for $e / \mu$ for each tower at two different energies.

- Calibration-II (at the end): 10 hours $2.5 \mathrm{k}$ events $(0.2 \mathrm{~h})$ for $e / \mu$ for each tower at one energy.

- Energy Scan: $=20$ hours

$10 \mathrm{k}$ events for $e / \pi / \mu$ at 10 energy points only at central injection.

- Position Scan: $=20$ hours

$10 \mathrm{k}$ events for $e / \pi / \mu$ at 14 injection points at one energy.

In addition, we assume 2 days and 1 day for beam tuning at the first and second configurations, respectively. In summary, we will need 5 days for the first test and 4 days for the second test. In order to change configuration from $8: 2$ to $12: 2$, about three days are needed with beam off. Therefore, we request nine full days of beam time and three days for re-configuration time. The schedule is shown in Table 2.

\section{Request for Support}

The following supports are necessary from Fermilab:

1. Preparation area: In order to unload, unpack, and check the delivered components, working area of about $5 \mathrm{~m} \times 5 \mathrm{~m}$ is necessary. A crane is necessary to directly move the equipments from the working area to the beam line. 
Table 2: Beam test schedule

\begin{tabular}{ll}
\hline \hline Period & August-September 1999 \\
Place & Meson Det. Bldg., Test Beam Area E-741 \\
Schedule & Early in August; Equipments arrive at FNAL. \\
& Mid of August; Component check. \\
& End of August; Set-up on the beam line. \\
& Early September to Late September; Beam test. \\
& 5-days beam time for the first configuration (8:2 volume ratio). \\
& 3-days of beam-off time to re-configure the module. \\
& 4-days beam time for the second configuration (12:2 volume ratio). \\
& End of September; Disassembling the setup on the beam line. \\
& Early October; re-packing and sending back to Japan. \\
\hline \hline
\end{tabular}

2. Crane operators: The calorimeter module consists of heavy lead plates. In order to assemble/re-configure the module, crane operation is required. We thus would like to request some crane operators for this work: for one day to unload delivered equipments, one week during assembling period, 3-days for re-configuring period, and one week for disassembling period.

3. Drift chambers for beam tracking and Cerenkov counters: In order to define incoming particles, we would like to use drift chambers and Cerenkov counters which are expected to be equipped in the beam line. We will also borrow related equipemts such as pre-amps, discriminators, TDCs, HV supplies, read-out cables, and gas systems.

4. Counting Room: In order to set up DAQ system well before the beam test, we would like to use a counting room near the test beam area from mid-August to the end of September.

5. Network Resources: In order to connect DAQ PC's to a network, we would like to have two IP addresses from mid-August to the end of September.

6. Electronic modules: We are planning to construct our DAQ system with PCs, NIM, CAMAC and FastBus. The PCs will be brought from Japan with DAQ softwares in them. We would like to borrow NIM/CAMAC/FastBus modules from FNAL module pool. At present, we would like to borrow followings.

- PMT HV supply $\times 200 \mathrm{ch}$,

- FastBus ADC (15bit eq.) $\times 180 \mathrm{ch}$,

- FastBus TDC $\times$ (necessary amout for beam-define drift chambers),

- CAMAC TDC $\times 16 \mathrm{ch}$,

- CAMAC Interrupt Register, Scalers, etc, and 
- Various NIM modules necessary to build trigger logic.

Detailed module list will be submitted later.

7. Cables: We would like to borrow cables from detectors to the counting room as follows.

- Signal cables $(\mathrm{BNC}) \times 200 \mathrm{ch}$; necessary length from the test area to the counting room.

- $\mathrm{HV}$ (SHV) $\times 200 \mathrm{ch}$; necessary length from the test area to the counting room.

- Drift chamber cables; necessary channels and length from the beam line to the counting room.

8. Other Instruments: We would also like to borrow following equipments.

Theodolites and levels for detector alignment.

Air conditioners for temperature control.

\section{Financial Arrangement}

Financial resources for this beam test is mainly from the JAPAN-US collaboration for High Energy Physics. We expect that our proposal on this test will be accepted for JFY1999. Most of the travel costs are provided by this resource. Another financial resource from KEK in JFY1999 is a KEK-Universities R\&D collaborations. Transportation costs are covered by these JAPAN-US and KEK R\&D budgets.

We therefore do not need to request FNAL for any financial supports for this beam test.

\section{Hazardous Material Treatment}

The lead plates are chemically dangerous material. Rude handling would generate/scatter lead powders, and thus could cause poisoning. However in out beam test, handling of lead plates will always be done using a crane. This accordingly provides very careful and gentle handling. Therefore the risk of generating lead powder is minimal for our beam test.

Gloves shall be used to handle the lead plates so as not to touch the lead plates directly. 


\title{
Appendix: List of the collaboration members
}

\author{
Table 3: Collaboration Members
}

\begin{tabular}{lll}
\hline \hline Tohru Takeshita & Shinshu Univ. & (Collaboration Representative ; Japan-side) \\
Teruki Kamon & Texas A\&M Univ. & (Collaboration Representative ; US-side) \\
Yoshiaki Fujii & KEK & \\
Jun'ichi Kanzaki & KEK & \\
Kiyotomo Kawagoe & Kobe Univ. & \\
Naoko Kanaya & Kobe Univ. & \\
Yoshiyuki Sugimoto & Kobe Univ. \\
Akira Takeuchi & Kobe Univ. \\
Fumiyoshi Kajino & Konan Univ. \\
Keitaro Furukawa & Shinshu Univ. \\
Shin Hong Kim & Uinv. of Tsukuba \\
Takashi Asakawa & Uinv. of Tsukuba \\
Ryutaro Oishi & Uinv. of Tsukuba \\
Atsuko Nakagawa & Uinv. of Tsukuba \\
Tatsuro Ohta & Uinv. of Tsukuba \\
Satoru Uozumi & Uinv. of Tsukuba \\
Katsushi Arisaka & UCLA \\
\hline \hline
\end{tabular}

